\title{
artigo
}

\section{A mulher com transtorno mental vítima de violência por parceiro íntimo: revisão integrativa}

\author{
Women with mental disorder victim to violence by an intimate partner: an integrative review \\ Mujeres con trastorno mental víctima de violencia por pareja íntima: revisión integradora
}

\section{RESUMO}

Objetivo: Analisar na literatura o atendimento às mulheres portadoras de transtornos mentais vítimas de violência por parceiro íntimo na Rede de Atenção Psicossocial. Metodologia: Revisão integrativa realizada nas bases MEDLINE, LILACS, SCiELO e BDENF através do cruzamento de 6 descritores em 3 idiomas. Resultados: Foram identificadas 10.579 produções primárias e incluídos 3 artigos segundo critérios de inclusão. Evidenciou-se a inexistência de protocolos e desqualificação técnica dos profissionais para essa demanda. Conclusão: 0 fomento a pesquisas sobre essa temática contribuirá para a qualificação profissional e para superação da invisibilidade das mulheres vítimas de violência com transtorno mental.

DESCRITORES: Violência contra a mulher; Violência por parceiro íntimo; Maus-tratos conjugais; Transtornos mentais; Serviços de saúde mental.

\section{ABSTRACT}

Aim: To analyze the literature about care provided to women with mental disorders who are victims of intimate partner violence in the Psychosocial Care Network. Methodology: Integrative review performed on the MEDLINE, LILACS, SCiELO and BDENF databases by crossing 6 descriptors in 3 languages. Results: 10,579 primary productions were identified and 3 articles were included according to inclusion criteria. The lack of protocols and technical disqualification of professionals for this demand was evidenced. Conclusion: The promotion of research on this theme will contribute to professional qualification and to overcome the invisibility of women victims of violence with mental disorders.

DESCRIPTORS: Violence against women; Intimate partner violence; Spouse abuse; Mental disorders; Mental health services.

\section{RESUMEN}

Objetivo: Analizar en la literatura la atención a mujeres con trastornos mentales víctimas de violencia de pareja en la Red de Atención Psicosocial. Metodología: Revisión integrativa realizada en las bases de datos MEDLINE, LILACS, SCiELO y BDENF cruzando 6 descriptores en 3 idiomas. Resultados: se identificaron 10.579 producciones primarias y se incluyeron 3 artículos según criterios de inclusión. Se evidenció la falta de protocolos y la descalificación técnica de los profesionales para esta demanda. Conclusión: La promoción de la investigación sobre este tema contribuirá a la calificación profesional y a superar la invisibilidad de las mujeres víctimas de violencia con transtorno mental.

DESCRIPTORES: Violencia contra la mujer; Violencia de pareja; Maltrato conyugal; Trastornos mentales; Servicios de salud mental.

RECEBIDO EM: 25/08/2020 APROVADO EM: 17/09/2020

\section{Pedro Camilo Calado da Silva}

Estudante do Curso de Bacharelado em Enfermagem, Instituto Federal de Pernambuco, Campus Pesqueira, Bolsista do Programa de Iniciação Acadêmica - FACEPE,

ORCID: 0000-0003-0817-0541

\section{Crisllayne Ohanna do Nascimento Pereira}

Estudante do Curso de Bacharelado em Enfermagem, Instituto Federal de Pernambuco, Campus Pesqueira, Bolsista do Programa de Iniciação Acadêmica - IFPE

ORCID: 0000-0001-6123-620x 


\section{Robervam de Moura Pedroza}

Bacharel em Enfermagem, Especialista em Gestão de Serviços e Sistemas de Saúde, Mestre em Saúde da Família, Professor e Coordenador do Curso de Bacharelado em Enfermagem, Instituto Federal de Pernambuco, Campus Pesqueira.

ORCID: 0000-0003-4771-3916

\section{Silvana Cavalcanti dos Santos}

Bacharel em Enfermagem, Especialista e Mestre em Saúde Pública, Doutoranda em Gestão e Economia da Saúde, Professora do Curso de Bacharelado em Enfermagem, Instituto Federal de Pernambuco, Campus Pesqueira.

ORCID: 0000-0002-6649-0423

\section{Valdirene Pereira da Silva Carvalho}

Bacharel em Enfermagem, Especialista em Saúde Pública, Mestre em Gestão e Economia da Saúde, Doutoranda em Ciências da Saúde, Professora do Curso de Bacharelado em Enfermagem, Instituto Federal de Pernambuco, Campus Pesqueira.

ORCID: 0000-0003-1296-2154

\section{Valquíria Farias Bezerra Barbosa}

Bacharel em Enfermagem, Especialista em Educação Profissional e Tecnológica-Área Saúde, Doutora em Ciências Humanas, Líder do Grupo de Pesquisa Cuidado e Promoção à Saúde, Professora do Curso de Bacharelado em Enfermagem, Instituto Federal de Pernambuco, Campus Pesqueira e do Mestrado Profissional em Educação Profissional e Tecnológica (PROFEPT), Instituto Federal de Pernambuco, Campus Olinda.

ORCID: 0000-0002-8200-2274

\section{INTRODUÇÃO}

A violência contra a mulher é devastadora por seus impactos físicos, emocionais e psicológicos a suas vítimas. Trata-se de um problema de saúde pública que requer investimento em pesquisas que subsidiem políticas e ações de prevenção primária, secundária e terciária. Requer estratégias de mapeamento da violência, suas formas, seus agentes e proporções para o estabelecimento de medidas protetivas e de assistência ${ }^{(1)}$.

A Lei $n^{\circ}$ 10.778/2003 estabeleceu a notificação compulsória em todo território nacional, dos casos de violência contra a mulher atendida em serviços de saúde públicos ou privados. Em complementaridade a Lei 11.340/2006, conhecida como Lei Maria da Penha, representou importante avanço ao ampliar a abrangência para todas e quaisquer ações ou condutas, baseadas no gênero, que causem morte, dano ou sofrimento, incluídas as violências física, psicológica, sexual, patrimonial e moral ${ }^{(2-3)}$.

A efetividade do direito das mulheres vítimas de violências à saúde e proteção social depende dos esforços tanto dos que formulam as leis e as fiscalizam, quanto dos que prestam atendimento direto. Portanto, é necessário que os profissionais de saúde que fazem o atendimento inicial a essas mulhe- res, mediante a escuta qualificada, consigam identificar esse agravo a saúde, acolhê-las em sua singularidade e informar os órgãos de proteção que compóem a rede intersetorial e seus horários de funcionamento ${ }^{(4)}$.

\section{A violência}

contra a mulher é

devastadora por

seus impactos

físicos, emocionais

e psicológicos a

suas vítimas.
No Brasil, as mulheres vítimas de violência por parceiro íntimo (VPI) portadoras de transtorno mental (TM) atendidas na Rede de Atenção Psicossocial (RAPS) estão expostas a múltiplas vulnerabilidades e estigmas relacionados à sua raça, grau de escolaridade e nível socioeconômico. Sendo assim, a discussão da VPI se faz necessária para o enfrentamento da violência contra as mulheres enquanto demanda da RAPS na lógica interdisciplinar e da clínica ampliada ${ }^{(5)}$.

O objetivo do presente artigo é analisar na literatura o atendimento às mulheres portadoras de transtornos mentais vítimas de violência por parceiro íntimo na Rede de Atenção Psicossocial.

\section{MÉTODO}

Trata-se de uma revisão integrativa de literatura desenvolvida segundo as etapas: definição do problema; estabelecimento dos critérios de inclusão e exclusão; identificação dos estudos selecionados; análise dos estudos que constituem a amostra; apresentação da revisão e síntese do conhecimento ${ }^{(6)}$.

A questão de pesquisa delineada segundo a estratégia PICO ${ }^{(7)}$ foi: Como a RAPS tem se organizado para o cuidado às mulheres portadoras de TM vítimas de VPI?

O critério para inclusão dos estudos foi: artigos que fizessem referência ao atendi- 


\section{artigo}

Calado da Silva, P.C.; Pereira, C.O.N.; Pedroza, R.M.; Santos, S.C.; Carvalho, V.P.S.; Barbosa, V.F.B.

A mulher com transtorno mental vítima de violência por parceiro íntimo: revisão integrativa

mento na RAPS a mulheres adultas com TM que sofreram VPI, publicados entre 2011 a 2019, nos idiomas português, inglês ou espanhol. Foram excluídos artigos que abordassem violência contra crianças, adolescentes, idosas, estudos secundários ou cartas ao editor, anais de eventos científicos, teses, dissertações, monografias e estudos duplicados.

Os artigos foram obtidos pelo cruzamento pareado dos Descritores em Ciências da Saúde: "violência contra a mulher", "violência por parceiro íntimo", "maus-tra-

Figura 1. Seleção dos Artigos da Revisão Integrativa, Pesqueira, PE, Brasil, 2019

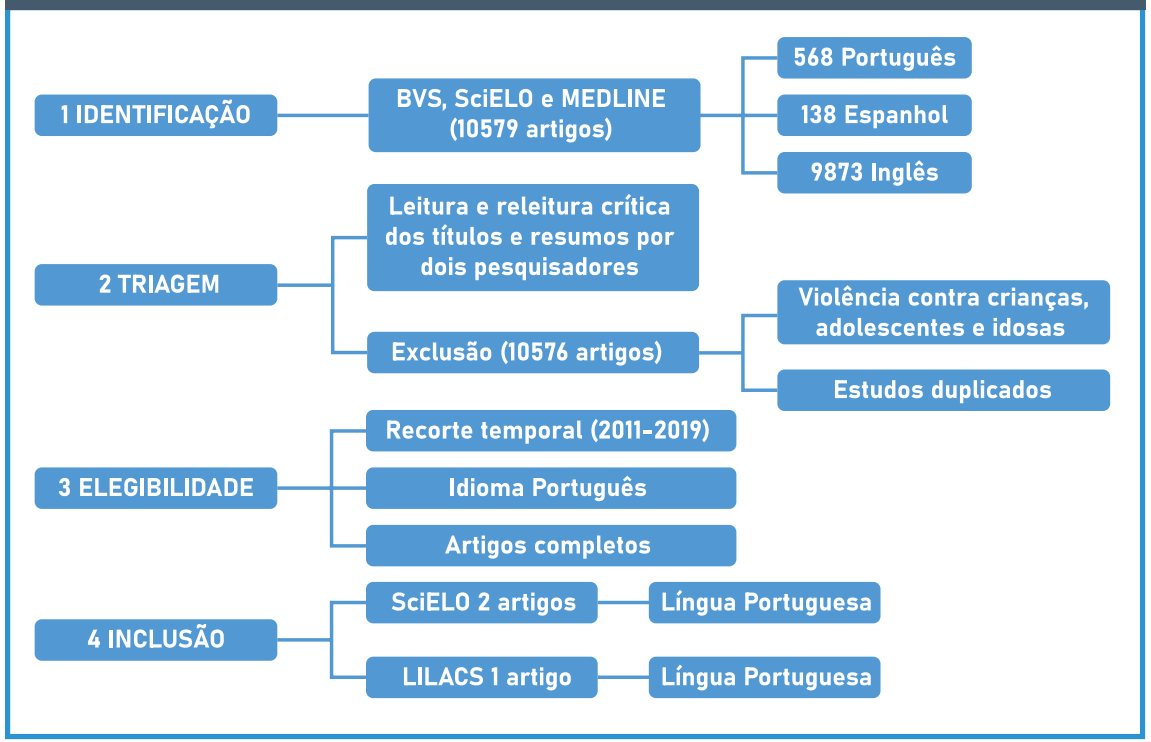

Fonte: Adaptação do Flow Diagram ${ }^{(9)}$

tos conjugais", "transtornos mentais", "desinstitucionalização" e "serviços de saúde mental", utilizando o operador booleano "AND", nas línguas portuguesa, inglesa e espanhola, nos bancos de dados: Scientific Electronic Library Online(SciELO), Biblioteca Virtual em Saúde(BVS), Sistema Online de Busca e Análise de Literatura Médica(MEDLINE).

A análise do nível de evidência dos artigos seguiu o método sugerido por Stillwell et $\mathrm{al}^{(8)}$.

Violência contra crianças,

Estudos duplicados

\section{Quadro 1. Caracterização dos estudos nacionais sobre o atendimento a mulheres com TM vítimas de VPI na RAPS (n=03), 2019.}

\begin{tabular}{|c|l|c|l|}
\hline ARTIGo & \multicolumn{1}{|c|}{ TítULO } & REVISTA/ANo & \multicolumn{1}{c|}{ OBJETIVO } \\
\hline 1 & $\begin{array}{l}\text { Mulheres, violência e atenção em saúde } \\
\text { mental: questões para (re)pensar o aco- } \\
\text { Ihimento no cotidiano dos serviços }\end{array}$ & $\begin{array}{c}\text { Avances en Psicología } \\
\text { Latinoamericana } \\
2014\end{array}$ & $\begin{array}{l}\text { Conhecer o acolhimento e atenção direcionados às } \\
\text { mulheres com demandas em saúde mental nos } \\
\text { serviços especializados para o enfrentamento da } \\
\text { violência contra as mulheres e na rede de atenção } \\
\text { psicossocial e hospitalar no município de Natal/RN. }\end{array}$ \\
\hline 2 & $\begin{array}{l}\text { Não é uma rede que flui - da invisibilidade } \\
\text { às possibilidades de novos modos de } \\
\text { cuidar: a violência contra as mulheres na } \\
\text { saúde mental }\end{array}$ & $\begin{array}{l}\text { Barbarói } \\
2015\end{array}$ & $\begin{array}{l}\text { Refletir sobre a inserção da violência contra as mulhe- } \\
\text { res no campo da saúde }\end{array}$ \\
\hline 3 & $\begin{array}{l}\text { (In)visibilidade da violência contra as mu- } \\
\text { Iheres na saúde mental }\end{array}$ & $\begin{array}{l}\text { Psicologia: } \\
\text { Teoria } \\
\text { e Pesquisa } \\
2017\end{array}$ & $\begin{array}{l}\text { Realizar um levantamento acerca da percepção, cren- } \\
\text { ças e conhecimentos sobre violência contra as mu- } \\
\text { lheres e políticas públicas em profissionais de saúde } \\
\text { mental. }\end{array}$ \\
\hline
\end{tabular}

O processo de seleção dos artigos que compuseram o corpus dessa revisão integrativa está descrito na Figura 1, com base no Preferred Re-porting Items for Systematic Reviews and Meta-Analyses (PRISMA) ${ }^{(9)}$.

Os artigos selecionados são oriundos de pesquisas qualitativas desenvolvidas no Brasil, com nível de evidência VI, publicados em revistas com Qualis CAPES B.

\section{DISCUSSÃO}

As pesquisas foram conduzidas por mulheres com formação acadêmica em psicologia e pós graduação em nível de mestrado e doutorado, o que expressa a sua postura de sororidade.

Destaca-se a importância da realização de outros estudos com desenhos de pesquisa diversificados e nível de evidência mais elevado, considerando seu poder de generalização e seu potencial interpretativo da realidade ${ }^{(8)}$. Por outro lado, os estudos qualitativos trazem consigo a potencialidade de "dar voz" a sujeitos sociais invisibilizados desvelando os significados, simbolismos, percepções, representações psíquicas e sociais do fenômeno em estudo ${ }^{(10)}$.

$\mathrm{Na}$ análise crítica dos textos foram encontradas convergências sobre a invisibilidade do sofrimento das mulheres vítimas de VPI, evidenciada pelo fato dos profissionais 
evitarem "tocar no assunto" para tentar assim desviar da problematização da situação. A adoção de "atitudes indiferentes" revelou que essa violência é considerada uma situação privada, de cunho pessoal e íntimo ${ }^{(10-12)}$.

Os três estudos identificaram o desconhecimento das equipes técnicas da RAPS e dos serviços de enfrentamento à violência sobre as diretrizes do modelo de atenção psicossocial brasileiro e quanto aos dispositivos legais de proteção e assistência as mulheres ${ }^{(10-12)}$.

A percepção por parte dos profissionais de saúde do internamento involuntário como a única medida protetiva a mulher em situação de violência revelou ainda o processo de invalidação do sofrimento, assim como da vulnerabilização, culpabilização e punição da vítima pela violência sofrida ${ }^{(13)}$. Configura-se como violência institucional simbólica que reverbera no aprofundamento do sofrimento das mulheres com TM vitimadas pela $\mathrm{VPI}^{(10-12)}$.

A desqualificação dos profissionais de saúde para o acolhimento dessas mulheres, a identificação da violência, o encaminhamento e notificação dos casos, assim como a dificuldade em distinguir entre notificação e denuncia resulta na subnotificação de $\operatorname{casos}^{(11-13)}$. A negação quanto a incorporar ações de enfrentamento a violência contra as mulheres nas demandas dos serviços da RAPS caracteriza a "recusa tecnológica" em oferecer a usuária um atendimento qualificado segundo as ferramentas, tecnologias e recomendações do campo da saúde para violências de gênero ${ }^{(13-14)}$.

Ao contrário, são privilegiadas práticas intervencionistas limitadas em sua possibilidade de atender as necessidades das mulheres vítimas de VPI com TM, a exemplo da medicamentalização. A centralidade da intervenção medicamentosa no cuidado configura-se como um componente do processo de medicalização do sofrimento e naturalização das violências, em que as questões subjetivas e sociais são desconsideradas ${ }^{(15)}$. Esse fenômeno sócio-histórico repercute na atribuição de diagnósticos de TM e no crescente consumo de benzodiazepínicos e antidepressivos entre mulheres vítimas de violências ${ }^{(16)}$.

As múltiplas vulnerabilidades que atingem as mulheres vítimas de violências tra-

\section{O cuidado a s}

aúde mental

no modelo de

atenção psicossocial

requer um processo

contínuo de

repensar ações e

valores, indagando

percepçôes e

preconceitos do

profissional ante a

mulher vitimada

pela VPI. zem à tona a questão da biolegitimidade enquanto ferramenta de conquista, vindicação e validação de direitos e do acesso ao atendimento integral. É preciso validar políticas e ações programáticas que permitam a superação dessas vulnerabilidades assim como o reconhecimento e valorização dos seus direitos e interesses ${ }^{(18)}$.

As vulnerabilidades programáticas que impactam na oferta da atenção integral a saúde dessas mulheres caracterizam-se pela ausência e/ou insuficiência dos recursos necessários à proteção contra riscos a sua integridade e à garantia de seu bem-estar físico, social e mental, que devem ser providos pelo Estado ${ }^{(18)}$.

Para superação dessa vulnerabilidade faz-se necessário rever o papel da gestão, planejamento e avaliação das ações de estruturação e fortalecimento das redes de atenção e proteção social às mulheres ${ }^{(19)}$. O acolhimento deve ser efetivado como diretriz operacional nos serviços de saúde e atenção psicossocial, além de garantidos a acessibilidade universal e o cuidado integral ${ }^{(20)}$.

O cuidado a saúde mental no modelo de atenção psicossocial requer um processo contínuo de repensar ações e valores, indagando percepções e preconceitos do profissional ante a mulher vitimada pela VPI. Uma formação acadêmica alinhada a complexidade da clínica ampliada pode subsidiar a compreensão e atuação profissional em dispositivos de desinstitucionalização com enorme potência para a construção de novas estratégias de cuidado na RAPS ${ }^{(10)}$. A educação permanente é uma outra ferramenta promissora no apoio às equipes multiprofissionais na construção de estratégias de identificação e notificação das violências e de fluxos de articulação em rede ${ }^{(21)}$.

Quanto às limitações do estudo, não foram consideradas teses, dissertações e monografias não publicadas em periódicos científicos.

\section{CONCLUSÃO}

A presente pesquisa contribuiu para identificar a escassez na literatura de trabalhos sobre o atendimento às mulheres vítimas de VPI na RAPS. 


\section{artigo}

Calado da Silva, P.C.; Pereira, C.O.N.; Pedroza, R.M.; Santos, S.C.; Carvalho, V.P.S.; Barbosa, V.F.B.

A mulher com transtorno mental vítima de violência por parceiro íntimo: revisão integrativa

Os estudos analisados apontaram a desqualificação técnica profissional e dificuldades dos atores no acolhimento, na notificação e no encaminhamento desses casos. Essas lacunas programáticas permitem a medicalização do sofrimento, subnotificação de casos e a ocorrência de violência institucional e simbólica contra as usuárias. Os silenciamentos, desinteresse, não validação e/ou naturalização da violência e do sofrimento, assim como a internação psiquiátrica involuntária são práticas remanescentes do modelo psiquiátrico manicomial, justificadas pelo argumento de "proteger" a vítima.

O desconhecimento das leis que amparam as mulheres e estabelecem diretrizes para a condução do atendimento a situações de violência, somado à inexistência de protocolos técnicos institucionais resulta em atendimentos direcionados pelo senso comum, de modo intuitivo, sem base teórico-prática sólida.

A capacitação dos profissionais na abordagem das mulheres vítimas de VPI e outras violências é caminho de excelência para provocar novos olhares e a construção de novas práticas de cuidado e de trabalho em rede, maximizando suas potencialidades.

\section{REFERÊNCIAS}

1. Oliveira FS, Araújo LM, Silva LL, Crispim ZM, Lucindo VBDB, OIiveira LN. Violência Doméstica e Sexual Contra a Mulher: Revisão Integrativa. HOLOS, 2017, v. 8(sn): 275-284. DOI: 10.15628/holos.2017.1903

2. Brasil. Lei $n^{\circ} 10.778$, de 24 de novembro de 2003. Estabelece a notificação compulsória, no território nacional, do caso de violência contra a mulher que for atendida em serviços de saúde públicos ou privados. Diário Oficial da União. Brasília (DF): 2003 24 nov.

3. Brasil. Lei Maria da Penha e Legislação Correlata. Brasília (DF): Senado Federal,

Subsecretaria de Edições Técnicas, 2011.

4. Pedrosa M, Zanello V. (In)visibility of violence against women in mental health. Psic.: Teor. e Pesq. 2017, 32(spe). DOI: 10.1590/0102-3772e32ne214

5. Brasil. Ministério da Saúde. Portaria $n^{\circ} 3.088$, de 23 de dezembro de 2011. Institui a Rede de Atenção Psicossocial para pessoas com sofrimento ou transtorno mental e com necessidades decorrentes do uso de crack, álcool e outras drogas, no âmbito do Sistema Único de Saúde (SUS). Diário Oficial da União. Brasília, DF; 2011.

6. Botelho LLR, Cunha CCA, Macedo M. O Método da Revisão Integrativa nos Estudos Organizacionais. Gestão e Sociedade.2011, 5(11): 121-136 DOI: 10.21171/ges.v5i11.1220

7. Joanna Briggs Institute. Reviewers' manual. Adelaide: JBI; 2014. Disponivel em: https://nursing.Isuhsc.edu/JBI/docs/ReviewersManuals/Economic.pdf.

8. Stillwell SB, Fineout-overholt E, Melnyk BM, Williamson KM. Evidence-based practice, step by step: searching for the evidence. Am J Nurs. 2010.110(1):51-3. DOI: 10.1097/01. NAJ.0000372071.24134.7e

9. Moher D, Liberati A, Tetzalaff J, Altman DG. Preferred reporting items for systematic review and meta-analysis protocols (PRISMA-P). PLoS Medicine. 2009;6(7):e1000097.

10. Barbosa LB, Dimenstein M, Leite JF. Women. Violence and mental Healthcare: Issues for (Re)thinking Reception in the Routine of Services. Av. Psicol. Latinoam. 2014. 32 (2). DOI: 10.12804/apl32.2.2014.09

11. Scisleski ACC, Maraschin C, Silva RN.Manicômio em circuito: Os percursos dos jovens e a internação psiquiátrica. Caderno de saúde pública. 2008. 24(2): 342-52. DOI: 10.1590/S0102$311 \times 2008000200013$

12. Meinhardt YM, Maia GF. Não é uma rede que flui - da invisibilidade às possibilidades de novos modos de cuidar: a violência contra as mulheres na saúde mental. Barbarói. 2015, 44 (esp): 120-136. DOI: 10.17058/barbaroi.v0i0.7440

13. Meneghel SN. Rotas críticas II: ferramentas para trabalhar com a violência de gênero. Santa Cruz do Sul: EDUNISC, 2009.

14.Tesser CD, Poli Neto P, Campos GWS. User embracement and social (de)medicalization: a challenge for the family health teams. Ciênc. saúde coletiva [online]. 2010, 15 (suppl.3): 36153624. DOI: 10.1590/S1413-81232010000900036

15. Maluf SW, Tornquist C. (org.). Gênero, saúde e aflição: políticas públicas, ativismo e experiências sociais. Florianópolis: Letras Contemporâneas, 2010. p. 21-67. DOI: 10.1590/S198464872012000600013

16. Rodrigues NO, Neri AL. Social, individual and programmatic vulnerability among the elderly in the community: data from the FIBRA Study conducted in Campinas, São Paulo, Brazil. Ciênc. saúde coletiva [online]. 2012, 17(8):2129-39. DOI: 10.1590/ S1413-81232012000800023

17. Mendes EV. As redes de atenção à saúde. Brasília: Organização Pan-Americana da Saúde, 2011.

18. Maluf SW, Biolegitimacy, rights and social policies: New biopolitical regimes in mental healthcare in Brazil. Vibrant, Virtual Braz. Anthr. [online]. 2015, 12(1):321-50. DOI: 10.1590/1809-43412015v12n1p321

19. Santos IMV, Santos AM. The relationship between healthcare personnel and patients (user acceptance) regarding the family healthcare program: a review of approaches in Brazilian journals. Rev. Salud Pública. 2011, 13(4):703-716. DOI: 10.1590/S012400642011000400015

20. Emerich BF, Onocko-campos R. Mental Health professional education: reflections based on the conceptions of Subject, Collective and Institution. Interface (Botucatu), 2019, 23 (sn). DOI: 10.1590/interface.170521

21. Gomes NP, Erdmann AL, Bettinelli LA, Higashi GDC, Carneiro JB, Diniz NMF. Significado de la formación profesional para el cuidado de la mujer en la violencia conyugal. Esc. Anna Nery, 2013, 17(4): 683-89. DOI: 10.5935/1414-8145.20130012 